\title{
Research on Train Wheel Diameter Correction Based on Multi - sensor Fusion and Gray - Scale Prediction
}

\author{
Teng Zhong and Shenghua Dai \\ School of Electronic and Information Engineering, Beijing Jiaotong University, Beijing, China
}

\begin{abstract}
Multi-sensor fusion is an effective way to realize low-cost and high-precision positioning on train. And Kalman filter algorithm is easy to be applied in the computer, so it is one of the important research directions of information fusion. This paper studies on the odometer in multi-sensor fusion system and analysis of its positioning error source. Based on the fuzzy adaptive Kalman filter algorithm, the gray level prediction model is introduced and improved, and the two methods are combined to improve the real-time and autonomy of the wheel diameter correction work. Verification of measured data and results of simulation demonstrate that the proposed algorithm provides high precision, high system efficiency and improved independent performance and it is of some value in application.
\end{abstract}

Keywords- multi-sensor; train positioning; gray prediction; Kalman filtering

\section{INTRODUCTION}

With the continuous development of China's economy and the construction of urbanization continues to deepen, the state of urban transport network of continuous improvement and expansion of a higher level of requirements. As of the end of 2016, more than 30 cities have built urban rail transit routes, and the total number of lines is 134.Urban rail transit systems must meet the requirements of being safe. The safe and accurate operation of the train is the real-time and accurate train operation control system. How to get real-time, accurate speed and location information on the train is critical, which is an important condition for improving rail transport efficiency and traffic safety [1].

Due to the particularity of its environment in urban rail transit, including Intensive buildings and serious signal interference, and from the cost point of view, the main vehicle locating sensors commonly used in urban rail transit trains are odometer, with other sensors to assist ${ }^{[2]}$.

With the continuous development of train operation technology, the use of multi-sensor information fusion to achieve the target carrier real-time high-precision dynamic positioning has been widely used ${ }^{[3]}$.In this paper, Kalman filter theory is combined with gray prediction to correct the train wheel error.

\section{PRINCIPLE AND ERROR ANALYSIS OF ODOMETER}

Using Pulse signal which could be got from a wheel speed sensor mounted on a train wheel could determine the speed of the train .The principle is as follows: When the installation is complete and parameter setting is also completed, the number of pulses is determined while the wheels turn around. Setting a fixed cycle to count the number of pulses could calculate the distance that the train had traveled. The formula is as follows:

$$
l=\frac{n \bullet \pi \bullet d}{N}
$$

$l$ : The distance traveled by train in a cycle

$n$ : The number of pulses received in a cycle

$d$ : Current wheel diameter

$N$ : The number of pulses that the wheel rotates normally for one week

The error in the odometer positioning comes mainly from count error due to idling and taxiing and wheel error due to wear and tear. In this paper, wheel error caused by wheel wear is studied.

\section{WHEEL DIAMETER CALIBRATION}

To deal with the error of the odometer due to the wear and tear, predecessors had made a lot of wheel diameter calibration program and made some sense .But there are some flaws.

\section{A. Balise}

When the train passes through the transponder, the train can get accurate positioning information. When the train passes through two adjacent transponders, the train can get accurate distance information. So it is easy to get the wheel diameter by the following formula.

$$
D=\frac{S_{\Delta b} \times N}{n_{\Delta b} \times \pi}
$$

$$
\begin{aligned}
& S_{\Delta b} \text { : The exact distance between adjacent balises. } \\
& n_{\Delta b} \text { : The number of pulses between the two balises. }
\end{aligned}
$$

But correcting the wheel diameter by balise exits the following defects.

One: only offer fixed-point correction wheel diameter value

Two: Need a large number of balises and increase the cost including construction and maintenance 
Three: need to be combined with the wheel speed sensor to complete the wheel diameter correction. When the wheel speed sensor break, the system can not work.

\section{B. Gray Prediction}

Gray theory $\operatorname{GM}(1,1)$ has advantage including less demand data, simple principle, and simple operation and so on. Literature correct the wheel diameter by the gray theory and achieve a higher accuracy. The mathematical model is as follows.

$X^{(0)}$ is the non-negative sequence and Accumulate it to get the 1 -AGO sequence called $X^{(1)}$.

$$
\begin{aligned}
& X^{(1)}=\left\{x^{(1)}(1), x^{(1)}(1), \ldots, x^{(1)}(n)\right\} \\
& x^{(1)}(k)=\sum_{i=1}^{k} x^{(0)}(i) ; k=1,2, \ldots, n
\end{aligned}
$$

By the $X^{(1)}$ could get the immediate sequence of values called $Z^{(1)}$.

$$
\begin{aligned}
& z^{(1)}(k)=\frac{x^{(1)}(k)+x^{(1)}(k-1)}{2} ; \\
& k=2,3, \ldots, n
\end{aligned}
$$

According to $\mathrm{GM}(1,1)$, set up gray differential equation as follows.

$$
x^{(0)}(k)+a z^{(1)}(k)=b
$$

$a$ : Development coefficient (Development of the number of gray), reflecting the development trend of the two sequences;

$b$ : Gray work volume, reflecting the relationship between the data changes.

Its whitening equation is as follows.

$$
d x^{(1)} / d t+a x^{(1)}=b
$$

Two parameters in the equation can be solved by the least squares method as follows.

$$
\hat{m}=[a, b]^{T}=\left(B^{T} B\right)^{-1} B^{T} Y
$$

$$
\begin{aligned}
& \text { Where, } Y=\left[\begin{array}{llll}
x^{(0)}(2) & x^{(0)}(3) & \cdots & x^{(0)}(n)
\end{array}\right]^{T} \\
& B=\left[\begin{array}{cc}
-z^{(1)}(2) & 1 \\
-z^{(1)}(3) & 1 \\
\mathrm{M} & \mathrm{M} \\
-z^{(1)}(n) & 1
\end{array}\right]
\end{aligned}
$$

The solution of the equation is based on the principle of minimum mean square error, where ensures the sum of variance to be minimal between $X^{(1)}$ and $\hat{X^{(1)}}$. By the formula four, it is easy to get the time response function of GM $(1,1)$ as follows.

$$
\hat{x^{(1)}}(k+1)=\left(x^{(0)}(1)-\frac{b}{a}\right) e^{-a k}+\frac{b}{a} \quad k=1,2, \ldots, n
$$

Through the formula (6), get the estimated sequence $\hat{X}^{(1)}$ and the estimated sequence called $\hat{X}^{(0)}$. Finally get the conclusion and complete the data forecast.

$$
\left\{\begin{array}{c}
\hat{x} x^{(0)}(1)=x^{(1)}(1) \\
\hat{x^{(0)}}(k+1)=x^{(1)}(\hat{k}+1)-x^{(1)}(k) \\
k=1,2, \ldots, n-1
\end{array}\right.
$$

Advantage:

One: More accurate estimate with less error

Two: Good independence and high degree of freedom

One: Disadvantage: exist relatively large cumulative error

Two: Poor real-time and cannot cope with the dramatic changes in the situation.

\section{Kalman Filter}

Multi-sensor information fusion is the development trend of high-precision train positioning, while the key is the Information fusion algorithm. It is possible and useful for discrete Kalman filter to apply to the computer. So it has been the attention of engineering applications. At present, Kalman filter theory is widely used in the field of train positioning based on multi-sensor information fusion.

Literature four propose a proposal to correct the wheel diameter by Kalman filter. Get real-time accurate distance information through Kalman filter and calculate the wheel diameter value by formula two.

\section{Advantage:}

One: Good real-time and real data

Two: High calibration accuracy

Disadvantage:

One: Computationally large

Two: The whole Kalman filter system is difficult to model and the system requires strict parameters

Three: need to be combined with the wheel speed sensor to complete the wheel diameter correction. When the wheel speed sensor break, the system cannot work. 


\section{RotATION CALIBRATION BASED ON KALMAN FILTER AND IMPROVED GRAY PREDICTION}

After analysis, it is easy to be proved that this is a good and useful program which is to combine with improved gray prediction and Kalman filter and take balise as a supplement.

In this way, the program will improve the accuracy of wheel diameter calibration by each module working together.

\section{A. Improved Gray Prediction}

Since the gray prediction GM $(1,1)$ is an exponential growth model based on the cumulative generation and the least squares method, the prediction accuracy depends on the structure of the model and the smoothness of the original sequence. Therefore, these two aspects can be optimized.

On the one hand, directly optimize parameter values. Using the following formula that the sequence $X^{(0)}$ is transformed into sequence $X_{1}^{(0)}$ to improve the smoothness of the sequence and reduce the value of $a$.

$$
f(x)=(x+1)^{1 / p}, p<0
$$

Finally, the sequence $X^{(0)}$ is obtained by the formula as follows.

$$
\hat{X^{(0)}}(k)=\left(\hat{X_{1}^{(0)}}\right)^{p}-1
$$

On the other hand, update parameter in time, which is called rolling prediction. Guarantee that the parameters can be more close to the real-time system in this way .Predicting after a period of time, Recalculate the parameters with the latest data to ensure that the model GM $(1,1)$ can be used for long-term prediction and reduce the cumulative error of the data.

\section{B. System Design}

Take the train mileage as a direct reference and regard the calibration of the wheel diameter as the "update - prediction update" cycle ${ }^{[4]}$.

The specific system program is as follows.

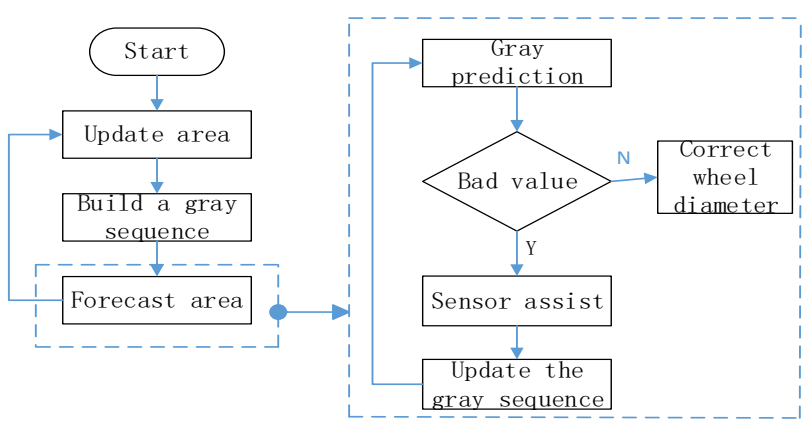

FIGURE I. WHEEL DIAMETERCALIBRATION PROGRAM
Enter the update area, where train passes through two adjacent balises, correct the wheel diameter by formula two if the odometer regular work.Simultaneously, Update the gray prediction model and update train information.

Enter the forecast area, where there is no balise, gray prediction plays a major role and Kalman wheel diameter calibration method plays a supervisory role.

Kalman filter system uses gray-scale prediction of the wheel diameter as a result of the input and output the train status information. When the odometer speed sensor is working properly and the detection unit detects that its output value is bad, it is proved that the gray prediction model has a large output error and the wheel diameter value has undergone dramatic changes. At this time, rely on Kalman filter wheel diameter correction method to provide several sets of new wheel prediction value as a new generation of gray prediction of the original sequence and continue to correct the wheel diameter value and verify the program as a comparison.

\section{SimUlation VERIFICATION}

\section{A. Improved Gray Prediction Model Validation}

This article uses the data inside the literature six and seven. The data get with the Beijing Railway Bureau three stores onsite experimental, which is persuasive. The simulation results are shown in the appendix.

After calculating, The relative residuals of the three predictive models are respectively: $0.7148 \%$ 、 $0.1645 \%$ 、 $0.093 \%$.This is very obvious that the program of this article further reduces the prediction error and improves the accuracy of the model GM $(1,1)$.

\section{B. Overall program validation}

This article investigated the $\mathrm{CRH} 2-06 \mathrm{C}$ new car appearance operation of about $400,000 \mathrm{~km}$ in the process of measuring the wheel diameter value with matlab for data fitting, which is as shown below.

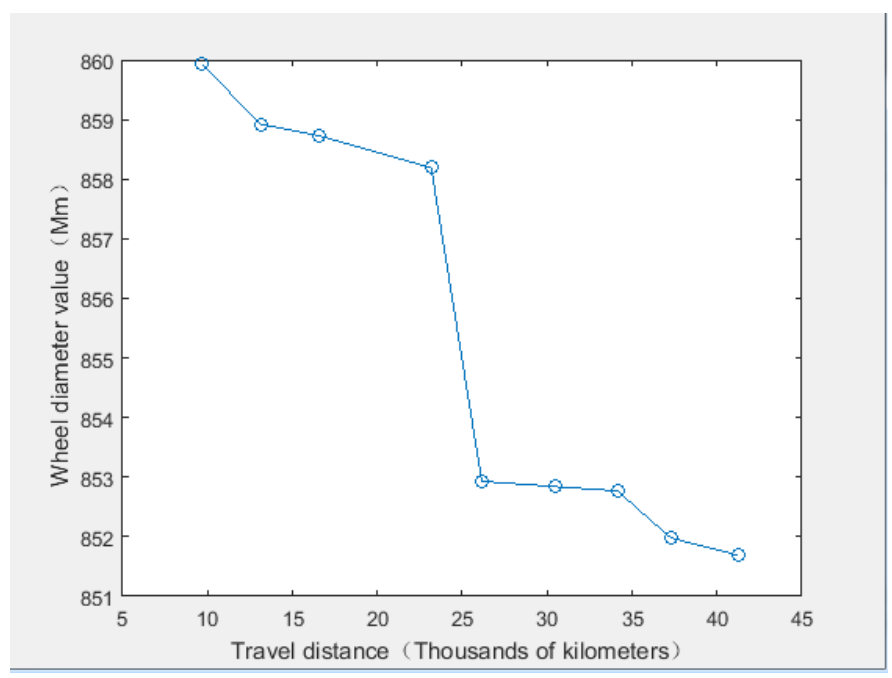

FIGURE II. WHEEL DIAMETER AND TRAVAL DISTANCE

As can be seen from the figure, the wheel diameter has changed over a certain period of time. In the range of turning 
points, it is necessary to reconstruct the original wheel diameter sequence. It is necessary to provide the discriminant basis and the new original sequence value based on the Kalman filter.

Due to the limited length of the experimental station, it cannot get enough positioning data. In order to verify the proposed rotation correction scheme, the simulation results are verified by matlab simulation data. Assume that the train is moving smoothly the system noise is superimposed on the acceleration and the variance array acceleration term is 0.1 .The initial wheel diameter is $267.38 \mathrm{~mm}$, the simulation time is $600 \mathrm{~s}$, the wear rate of the round wire is $0.0054 \mathrm{~mm} / \mathrm{s}$ in the first $300 \mathrm{~s}$, the wear rate of the inner diameter of 300 is $0.0108 \mathrm{~mm} / \mathrm{s}$ and the sampling period is $0.5 \mathrm{~s}$.

The error between the predicted value and the true value is the vertical axis, and the time is the horizontal axis. The resulting figure is shown below.

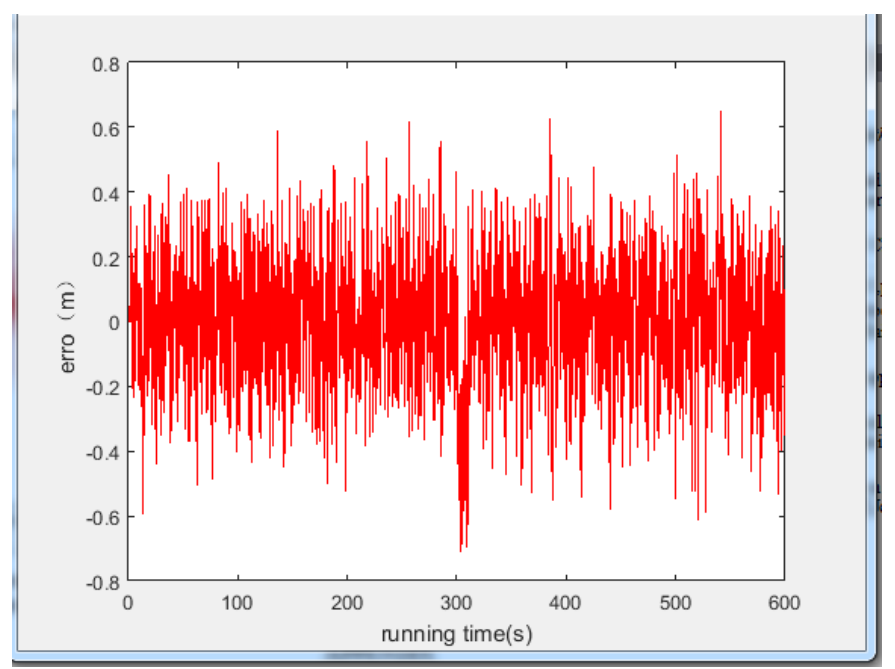

FIGURE III. PREDICTION ERROR AND RUNNING TIME
As can be seen from the figure, the error is relatively large, and then become stable during the period of 300s-320s.It is shown that the Kalman filter based axle method has played a role in monitoring and correcting the gray scale prediction model, so that the gray scale prediction model has better realtime and adaptability, and more suitable for the actual operation of the scene.

\section{CONCLUDING REMARKS}

This paper analyzes the odometer in the train positioning system and find the source of its positioning error. The error has been studied in depth which is caused by wheel abrasion.Combining the Kalman filter and the gray prediction model has not only improved the prediction accuracy but also reduced reliance on the sensor. From some point of view that the system reduce the amount of computing system and improve the autonomy of the system, this paper has a certain practical value.

\section{REFERENCE}

[1] Zhenxing Zhang,Research on Train Location Method in Urban Rail Transit, beijing university 2008

[2] Xinhui Wu,Xuan Cai,Hanqing, Fault Detection and Correction Method of Train Gauge Based on Multi - sensor,Research on Urban Rail Transit[J],May 2015,pp 19-25

[3] Xinhui Wu,Xuan Cai,Hanqing ,The Method of Checking the Wheel Length of Rail Transit Train Based on Multi - sensor Information Fusion,Research on Urban Rail Transit[J],June,2015,pp 21-27

[4] Peiding Liu,Rongwu Chen,Research on Velocity Measurement Algorithm Based on Speed Sensor,Railway Computer Applications[J],February 2016 ,pp 50-54

[5] Jun Zhang,Gray prediction model and its application [D],Xi'an University of Technology, 2008.

[6] Chao Cui, Yadong Zhang,Jin Guo,Improved gray scale prediction in train wheel diameter correction [J],Railway standard design,January 2016,pp $139-143$

[7] Jiang Liu,Bogen Cai,Jian Wang,Tao Tang,Study on calibration method of train wheel alignment based on gray theory [J],Journal of the China Railway Society,May 2011,[pp 54-59

APPENDIX

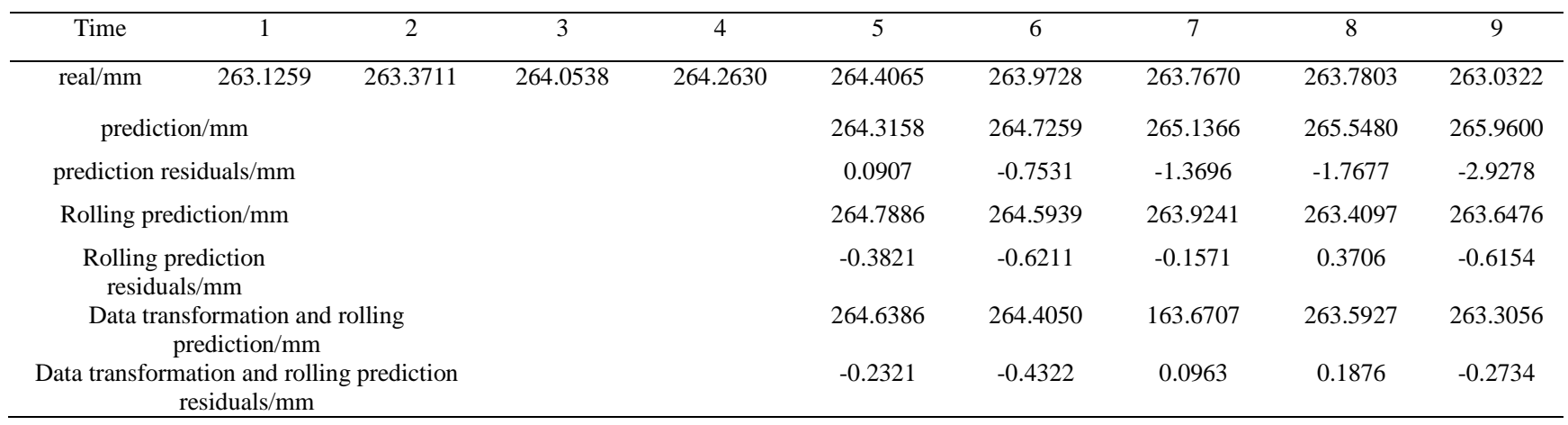

